\title{
Modeling interactions in ambient intelligence
}

\author{
Luis A. Castro ${ }^{1}$ (D) Jose Bravo $^{2}$ (D) \\ Accepted: 8 April 2021 / Published online: 16 April 2021 \\ (C) The Author(s), under exclusive licence to Springer-Verlag London Ltd., part of Springer Nature 2021
}

\section{Introduction and motivation}

Traditional notions of Human-Computer Interaction involve explicit interaction with users through input devices such as the keyboards or touchscreens, and output devices, such as computer displays or printers, that display information to users. Now, with the advent of ambient intelligence (AmI) interactions may also happen implicitly $[1,2]$ via sensors embedded in the environment that serve to mediate or articulate interaction using responsive technology. Despite previous research efforts, it is now with ever increasing number of sensors embedded in the environments (e.g., mobile, wearables, and pervasive technologies) and recent approaches (e.g., data analytics) that research in the area has been advancing in terms of better leveraging context. In this sense, many scenarios are increasingly feasible in which primarily implicit interactions between humans and the environments can be effectively mediated or articulated by responsive technology.

One of the main motivations of this special issue was inviting contributions that highlight the transition from conventional interaction methods (explicit) towards the new proposals for interacting in smart environments. In these kinds of contexts, the interaction disappears in the daily users' activities. In this context, the need to consider scenarios like those proposed in the AmI paradigm are highly encouraged. In these scenarios, the environment

Luis A. Castro

luis.castro@acm.org

Jose Bravo

Jose.Bravo@uclm.es

1 Department of Computing and Design, Sonora Institute of Technology (ITSON), 5 de Febrero 818 Sur, Centro, 85000 Ciudad Obregon, Sonora, Mexico

2 Department of Information Systems and Technologies, University of Castilla-La Mancha, Paseo de la Universidad 4, 13071 Ciudad Real, Spain and wearable sensors must be considered to the extent that, when combined with context awareness aspects, can make possible the involvement of users into intelligent interactions mechanisms [3].

\section{Submission and summary of contributions}

We launched an open call for papers in September 2019. We started receiving submissions in December 2019. The review process was somehow disrupted due to the COVID-19 pandemic, which caused additional workload for our reviewers and guest editors.

The review process was single-blind peer review. Each submission received three rigorous reviews from experts from the Americas, Europe, Asia, and Oceania. In the end, 9 manuscripts were accepted. The valuable support from our distinguished reviewers helped strengthen the manuscripts.

Some of the works aimed at supporting the work of designers and developers. First, the work entitled "User interface design patterns and ontology models for adaptive mobile applications" by Braham et al. [4] presents how design patterns and ontology models can be combined to creating adaptive mobile applications. The model enables the adaptation of mobile apps at runtime to different user needs, different context scenarios, interactive design modes or technology requirements. This work is evaluated by using three scenarios and a group of users who assessed the scenarios in terms usability.

Also, the work of Gil et al. [5] entitled "Modeling and smart prototyping human-in-the-loop interactions for AmI environments" presents a user-centered design method that defines a process with a set of tools and techniques to systematically design, prototype, and validate human-in-the-loop (HiL) solutions. In doing so, they aim at tackling the challenge of designing human involvement in AmI systems. The system was evaluated with 15 users, and the results suggest that the proposed method is more useful than traditional prototyping where designers observe the users interacting with a prototype and manually extract knowledge for refining the prototype. 
The third work by Silva-Rodríguez et al. [6] also facilitates the work of designers. The work "Predicting interaction design patterns for designing explicit interactions in ambient intelligence systems: a case study" presents the presents a model for inferring patterns of interaction design by processing text-based requirements. The predictive model is based on four classification algorithms: logistic regression, multinomial Naive Bayes, linear support vector machine, and random forest. The manuscript presents the performance results from the algorithms, and presents an evaluation performed with professional designers who rated the predictions of the model considering us usefulness and ease of use. The results from this work suggest that such algorithms can help professional designers make design decisions.

The fourth manuscript by Castells-Rufas et al. [7] is entitled "Continuous touch gesture recognition based on RNNs for capacitive proximity sensors." This work presents how RNNs can be used in embedded systems to process the data from low-cost capacitive sensors to recognize gestures as touches and slides in various scenarios including the use of gloves and bare hands. In this work, they introduce the use of Bayesian optimization with execution platform constraints to implement accurate gesture recognition sensors based on 1D capacitive sensor arrays. To validate the results, several RNN-based models are implemented and optimized for their execution on embedded automotive microcontrollers. The results of this work show that LSTM and GRU-based designs are adequate achieving an average recall of the gesture classes over $95 \%$.

The fifth aper, by Casillas-Figueroa et al. [8] entitled "ReminiScentia: shaping olfactory interaction in a personal space for multisensory stimulation therapy" presents an evaluation of the effectiveness of ReminiScentia in evoking brain responses like those generated by manually delivered scents. ReminiScentia is as an olfactory display to generate and deliver scents and was used to deliver the olfactory stimuli. From the results, user responses between manual deliver and ReminiScentia were comparable. The results of the study suggest that ReminiScentia is a reliable device to generate olfactory stimuli and measurable, which can be used to generate pleasurable experiences for users.

The sixth manuscript entitled "An Adaptive Model to support Biofeedback in AmI environments: A Case Study in Breathing Training for Autism" by Morales et al. [9] presents an adaptive model to support biofeedback taking user's physiological data, user's adherence to therapy, and environmental data to personalize its parameters and stimuli. In this work, they present EtherealBreathing, a biofeedback system designed to help children with autism practice box breathing. The model adapts the visual and audible stimuli of EtherealBreathing according to changes in each user's physiological data. Personalization is achieved through a series of variables that modify the behavior of the system, adapting it and adjusting it according to the user's performance.
The seventh work is entitled "Bringing proxemics to walker-assisted gait: using admittance control with spatial modulation to navigate in confined spaces" by Jimenez $\mathrm{t}$ al. [10]. This work presents a navigation strategy for a smart walker with social conventions defined by proxemics. In essence, they use an admittance controller to generate haptic and visual signals for a safe navigation within a corridor by considering how interactions take place in real-world settings. To validate this work, they carried out two experiments in which they first used a realistic environment, and then in a real environment. The results of this work enable a safe social navigation with a comfortable velocity of $0.244 \mathrm{~m} / \mathrm{s} \pm 0.1196$.

The eight manuscript by Alvarez et al. [11] present the work entitled "Applying the concept of implicit HCI to a groupware environment for teaching ethics", in which they report on an experience in which we added implicit HCI (iHCI) to an existing application that supports ethics education called EthicApp. EthicApp implements a methodology in which students read about a case that presents an ethical dilemma, report their personal position on it, and then debate and argue in groups of three members. Among two different configurations, empirical results show that heterogeneous grouping stimulates greater exchanges of argumentative discourse among the students, when they require to analyze their responses together. In turn, EthicApp was modified to automatically generate heterogeneous groups.

Finally, Finochietto et al. [12] present their work intitled "An IoT-based infrastructure to enhance self-evacuations in natural hazardous events." The manuscript proposes an IoTbased infrastructure that supports emergency evacuation of civilians, allowing people to participate as information providers and consumers. The system uses information such as people location and the status of the area to deploy breadcrumbs that guide people to shelters and safe places. He evaluation of the system included experimental data collection and a simulation, which yielded promising results.

Acknowledgements We thank the authors who really made this issue possible. Also, we want to thank the reviewers who provided valuable feedback to authors.

\section{References}

1. Schmidt A (2000) Implicit human computer interaction through context. Pers Technol 4(2):191-199

2. Schmidt A, Kranz M, Holleis P (2005) Interacting with the ubiquitous computer: towards embedding interaction. In: Proceedings of the 2005 joint conference on smart objects and ambient intelligence: innovative context-aware services: usages and technologies (pp. 147-152)

3. Kranz M, Holleis P, Schmidt A (2009) Embedded interaction: interacting with the internet of things. IEEE Internet Comput 14(2):46-53 
4. Braham A, Buendía F, Khemaja M, Gargouri F (2021) User interface design patterns and ontology models for adaptive mobile applications. Pers Ubiquitous Comput $1-17$

5. Gil M, Albert M, Fons J, Pelechano V (2021) Modeling and "smart" prototyping human-in-the-loop interactions for AmI environments. Pers Ubiquitous Comput 1-32

6. Silva-Rodríguez V, Nava-Muñoz SE, Castro LA, Martínez-Pérez FE, Pérez-González HG, Torres-Reyes F (2021) Predicting interaction design patterns for designing explicit interactions in ambient intelligence systems: a case study. Pers Ubiquit Comput 1-12

7. Castells-Rufas D, Borrego-Carazo J, Carrabina J, Naqui J, Biempica E (2020) Continuous touch gesture recognition based on RNNs for capacitive proximity sensors. Pers Ubiquitous Comput 1-18

8. Casillas-Figueroa R, Morán AL, Meza-Kubo V, RamírezFernández C, Acosta-Quiroz C, Orihuela-Espina F, MonteroHernandez S (2020) ReminiScentia: shaping olfactory interaction in a personal space for multisensory stimulation therapy. Pers Ubiquit Comput 1-17
9. Morales A, Cibrian FL, Castro LA, Tentori M (2021) An adaptive model to support biofeedback in AmI environments: a case study in breathing training for autism. Pers Ubiquit Comput 1-16

10. Jiménez MF, Scheidegger W, Mello RC, Bastos T, Frizera A (2021) Bringing proxemics to walker-assisted gait: using admittance control with spatial modulation to navigate in confined spaces. Pers Ubiquitous Comput 1-19

11. Alvarez C, Zurita G, Baloian N (2021) Applying the concept of implicit HCI to a groupware environment for teaching ethics. Pers Ubiquit Comput 1-19

12. Finochietto JM, Micheletto M, Eggly GM, Centelles RP, Santos R, Ochoa SF, ... \& Orozco J (2021) An IoT-based infrastructure to enhance self-evacuations in natural hazardous events. Pers Ubiquitous Comput 1-18

Publisher's note Springer Nature remains neutral with regard to jurisdictional claims in published maps and institutional affiliations. 